\title{
Reconstructions in Atomic and Electronic Structures at Gold-Oxide Interfaces
}

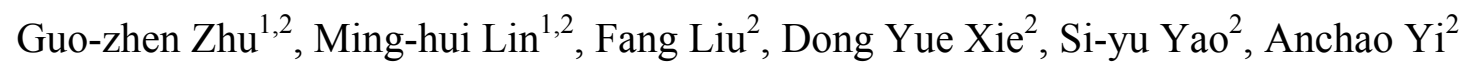

1. Department of Mechanical Engineering and Manitoba Institute for Materials, University of Manitoba, Winnipeg, MB, Canada

2. State Key Laboratory of Metal Matrix Composites, School of Materials Science and Engineering, Shanghai Jiao Tong University, Shanghai, P.R. China

The interaction between gold and oxides plays an important role in developing advanced materials for catalytic, photonic, and microelectronic applications. For example, $\mathrm{Au} / \mathrm{TiO}_{2}$ [1] and $\mathrm{Au} / \mathrm{MgAl}_{2} \mathrm{O}_{4}$ [2] show excellent catalytic activity and $\mathrm{Au} / \mathrm{CeO}_{2}$ [3] has tunable reaction selectivity for energy-related reactions. $\mathrm{Au} / \mathrm{SrTiO}_{3}$ interface [4] affects the tunneling probability, which is an important characteristic for device design. In spite of their technological importance, the microscopic nature of gold-oxide interfaces, primarily from the interaction between the delocalization of bonding electrons in gold and ionic bonds in oxides, is still a matter of debate.

Here, we studied structural characters of heat-treated gold-oxide interfaces. Gold particles supported on oxide single crystals were prepared using a thermal dewetting method. The 5-10nm gold overlayers were sputtered on clean surfaces of selected oxide substrates (MTI corp.). Gold overlayers break into particles during the heat treatment, which was carried out over a temperature range of $800-1100^{\circ} \mathrm{C}$ in high-purity Ar atmosphere. The cross-sectional samples were prepared by the Focused Ion Beam (FIB) technique. The atomic and electronic structures at interfaces were characterized using scanning transmission electron microscopy (STEM).

Atomic reconstructions at interfaces have been experimentally observed in a lattice-matched system of $\mathrm{Au} / \mathrm{MgAl}_{2} \mathrm{O}_{4}$ (spinel, (111)-oriented). [5-7] The gold lattice aligns with oxide substrates after the heat treatment at high temperatures. As shown in Fig.1, three different interfaces, including the one with direct adhesion of $\mathrm{Au}$ and $\mathrm{Al}(\mathrm{O})$ layer of $\mathrm{MgAl}_{2} \mathrm{O}_{4}$, monolayer, and two-layer interfaces, form over a temperature range up to the melting point of gold, according to experimental observation and theoretical prediction. The arrangement of gold atoms at interfaces are perfect $\{111\}_{\mathrm{Au}}$, atoms missing in the first $\{111\}_{\mathrm{Au}}$, and atoms mixing in the two adjacent atomic planes. Such atomic arrangements in the reconstructed interfaces show a strong similarity to the arrangement of metal cations in the spinel lattice. In corresponding to atomic reconstructions, bonding correlations are confirmed using electron energyloss spectroscopy. Within another lattice-matched system of Au-SrTiO 3 ((111)-oriented), only the interface, with direct adhesion of $\mathrm{Au}$ and $\mathrm{Ti}$ layer, is observed. (see Fig.2) Similar to the case of $\mathrm{Au} / \mathrm{MgAl}_{2} \mathrm{O}_{4}$, the inter-spacings between $\mathrm{Au}$ layer and topmost oxide layer are smaller than their bulk counterparts. Experimental inter-spacings agree with output values from the first-principle density function theory (DFT) calculations. With the increase in lattice mismatches, the interface has more complicated structures, which are related to the crystallographic orientations of gold particles on oxides. In the case of $\mathrm{Au}-\mathrm{TiO}_{2}$ (rutile, (110)-oriented), the interface includes a few monolayers, which have significant different atomic arrangements compared to the bulk lattices (see top image of Fig.3). The detailed interface structures and their thermodynamic conditions are currently being investigated in order to shed light on the understanding of two-dimensional phase transformation at gold-oxide interfaces. [8] 
References:

[1] M. Chen et al, Science 306(2004), p. 252.

[2] C. H. Christensen et al, Angew. Chem. Int. Ed. 45 (2006), p. 4648

[3] Y. Yamada et al., Nat. Chem. 3(2011), p. 372.

[4] R. Kraya et al, Nano Lett. 4(2010), p. 1224.

[5] G.-Z. Zhu et al, Appl. Phys. Lett. 105(2014), p. 231607.

[6] F. Liu et al., Mater. Charact. 113(2016), p. 67.

[7] F. Liu et al., Adv. Mater. Interface. 5(2018), p. 1701664.

[8] The authors acknowledge funding from the National Natural Science Foundation of China (No.51401124), the support from Canada Research Chair Program and University of Manitoba.
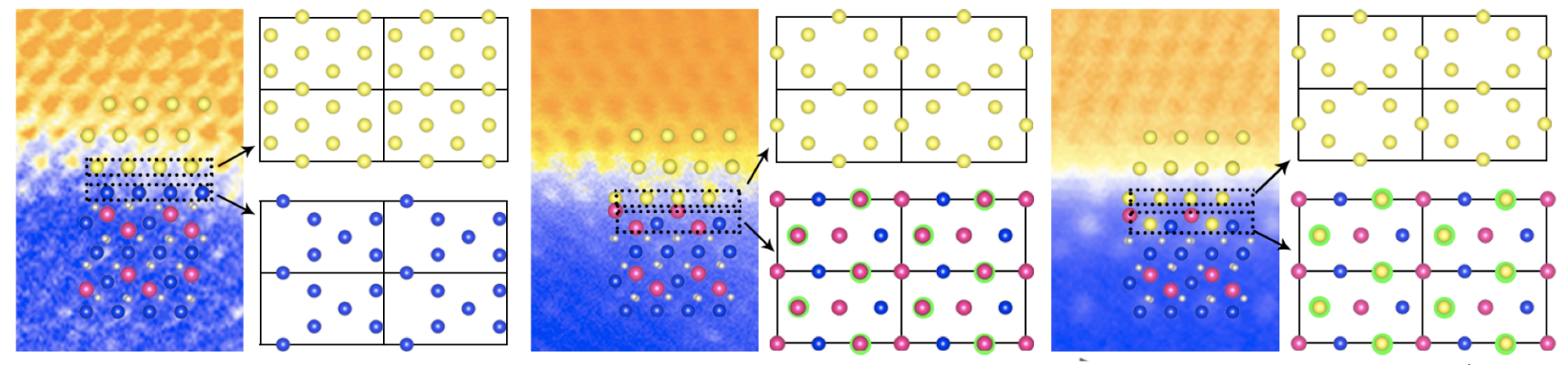

Temperature $\uparrow$

Figure 1. Color-coded STEM-HAADF images for reconstructed $\mathrm{Au} / \mathrm{MgAl}_{2} \mathrm{O}_{4}$ interfaces viewed from $<110>_{\mathrm{MgAl}_{2} \mathrm{O} 4}$. From left to right are the direct adhesion of $\mathrm{Au}$ and $\mathrm{Al}(\mathrm{O})$ layer of $\mathrm{MgAl}_{2} \mathrm{O}_{4}$, monolayerreconstructed interface, and two-layer interfaces. Insets are the atomic models simulated by firstprinciple density function theory (DFT) calculations. The yellow, blue, red, and gray spheres stand for $\mathrm{Au}, \mathrm{Al}, \mathrm{Mg}$, and $\mathrm{O}$, respectively. Beside each experimental image, the atomic arrangements of adjacent monolayers at interfaces are viewed along $<111>_{\mathrm{MgAl}_{2} \mathrm{O} 4}\left(/ /<111>_{\mathrm{Au}}\right)$. Green circles label these atoms with slightly different positions along the vertical direction.
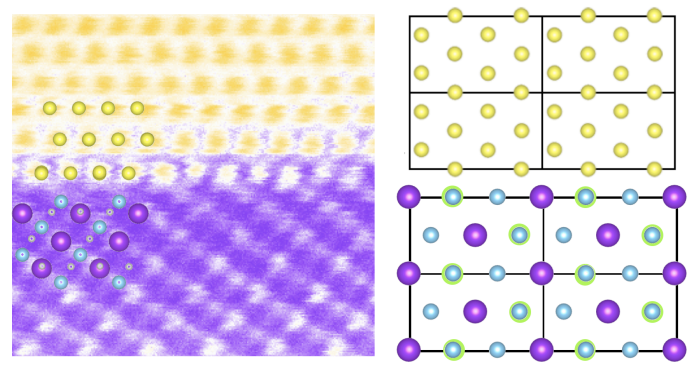

Figure 2. Left is the color-coded STEMHAADF image for $\mathrm{Au} / \mathrm{SrTiO}_{3}$ interface viewed from $<110>_{\mathrm{SrTiO} 3}$. Inset is the atomic model obtained by DFT calculations. The yellow, blue, purple, and gray spheres stand for $\mathrm{Au}, \mathrm{Ti}$, $\mathrm{Sr}$, and $\mathrm{O}$, respectively. Right is the atomic arrangements of adjacent monolayers at interfaces are viewed along $<111>{ }_{\mathrm{SrTiO3}}$ $\left(/ /<111>_{\mathrm{Au}}\right)$. Green circles label the second $\mathrm{Ti}$ layer from the interface.

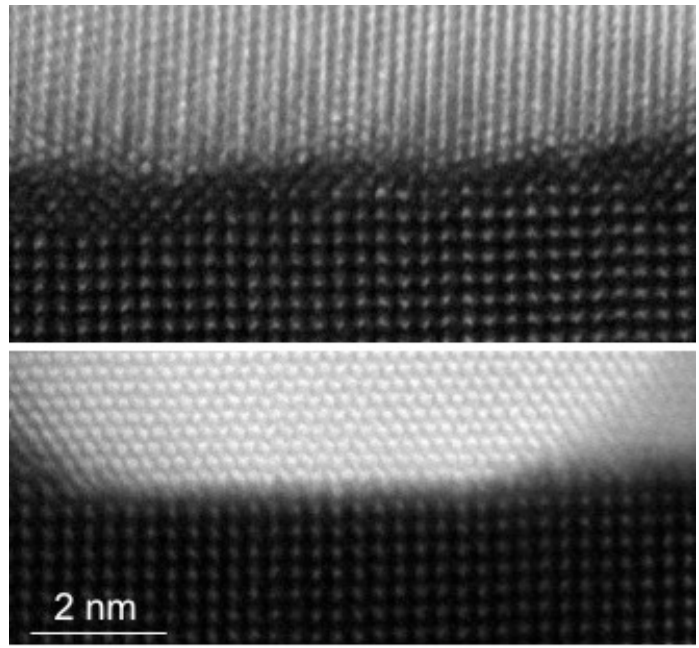

Figure 3. STEM-HAADF image for reconstructed $\mathrm{Au} / \mathrm{TiO}_{2}$ interfaces viewed from $<001>_{\mathrm{TiO} 2}$. (a) and (b) are reconstructed interfaces, detected with different oriented gold particles on top of $\{110\}_{\mathrm{TiO} 2}$. 